\title{
Application of Mobile Nursing Information System in High-quality Nursing
}

\author{
Xiu Qing Wang ${ }^{1, a^{*}}$, Ye Luo ${ }^{2}$, Min Shang ${ }^{2}$, Zhi Ru Li ${ }^{2}$, Min Zhang ${ }^{2}$ and Zhong \\ Ping Sun ${ }^{2}$ \\ ${ }^{1}$ Nursing department, The first affiliated hospital of Qiqihar Medical Univeristy, China \\ ${ }^{2}$ The first affiliated hospital of Qiqihar Medical Univeristy, China \\ aemail: wangxiuqing234@126.com
}

Keywords: Mobile nursing information system; High-quality nursing; Mobile nursing.

\begin{abstract}
With the development of information technology, the information system is applied in hospitals more and more widely. The mobile nursing information system is composed of nursing personnel and computers, which can be used to collect, store and process the information on nursing management and business technology. As it is one of important information systems in hospital, it is widely used in high quality nursing. In this paper, the application of mobile nursing information system in high quality nursing is described, and its advantages in this aspect are analyzed, and finally its application status in China is discussed.
\end{abstract}

\section{Introduction}

With the development of science and information technology, one of important development symbols for modernized hospital is the close combination of information technology and medical activities. As nursing is a major component of the hospital work, its information technology has been developed rapidly. The establishment and improvement of mobile nursing information system has led to the transformation of traditional model, which is of great significance to implement the patient-centered nursing concept and improve the nursing quality management in scientification and standardization. At present, on the initiative of the Ministry of Health, major hospitals attach more and more importance to the quality nursing services. The high quality nursing services have strict requirements on nurses in these aspects: service attitude, ability in solving problems of patients, work responsibility, proficiency in operation, patients' satisfaction. The core of high quality service is to return nurses to patients and return time to nurses, enabling nurses to be freed from the heavy amount of non-nursing work. At present, the mobile nursing information system has been applied in clinical nursing work in China's major hospitals, which aims at reducing the pressure on nurses, improving work efficiency, ensuring the demands for nursing excellence and making the society, doctors and patients satisfied.

\section{Mobile Nursing Information System}

The mobile nursing information system is the typical application of mobile computer technology in the hospital clinical nursing. Through the hand-held terminal of personal digital assistant (PDA), it can accomplish these tasks in the nursing work: bedside information collection, medical advice reminding, medical advice verifying and performing, patient identity verifying and nurse workload statistics, dramatically changing the model of traditional nursing work and relevant processes. For nursing measures, the real-time and real-name records should be ensured; for the bedside medical advice, the accuracy in verifying the identity should be ensured; the nursing work should enter the ward. Based on the hospital's HIS system, the mobile nursing information system uses the wireless scanning function of PDA to verify the valid information of patient at the bedside, taking the hospital information LAN as the wireless platform and wrist strap and barcode as the technological form. It can be used for direct information entry operation at the bedside relevant to medical order 
management, medical record management and vital signs, achieving the extension of nurses' station to the bedside of patient.

The mobile nursing information system has the following basic functions: identifying the patient's identity, retrieving and making the statistics of patients' information, recording the patient's nursing process, making the real-time collection of vital signs, calculation functions (including intake and output, body mass index, infusion drip, expected date of confinement, etc.), medical order query, implementation and statistics, barcode scanning test specimens, oral medication, transfusion labels, entry of consumables and charges, drug query(specifically, including information on dosage, side effects, drug interactions and drug price, etc.) and laboratory report query. For nurses, their priority selection is to use the mobile nursing information system to capture vital signs and drug reference information. With the wide application of mobile nursing information system, its functional scope will become wider and wider.

\section{Application of Mobile Nursing Information System in High Quality Nursing}

Management of Patients' Information. For the identification and verification of patient identity, the hospital generally uses a two-dimensional barcode wrist strap. When the patient handles the hospitalization procedure, the patient's barcode wrist strap is printed by the admission office in a unified way, in which the patient's name, gender, age, admission number and the number of two-dimensional bar code for scanning for identification are included. When the patient is accepted by the department, the nurse uses PDA to scan the wrist strap to check the patient's admission information. The PDA scanning time is the accurate admission time of patient. The nurse can scan the barcode and drug label on the wrist strap through using PDA to verify the drugs. If the information matches, the system automatically completes the checking program; if the information is incorrect, the system displays "not matching" and it will immediately makes sounds for alarm. By doing so, the errors caused by the nurses' fixed thinking of repeated checks can be avoided, and thus the occurrence of nursing errors can be prevented. When the patient's check results must be retrieved timely, the nurses can accurately find various checking results and other nursing information through PDA at the patient's bedside. This function allows the nurse to know more about the patient's disease more rapidly and communicate with the patient more effectively, and finally provide effective health guidance.

Collection and Entry of Patients' Information. The nurse can collect the data of vital signs at the bedside with PDA. The data are transmitted back to the PC terminal to automatically generate the single chart for human body temperatures. Then the system can automatically generate a list for patients required to check temperatures at the current time period based on the patient's body temperature and surgical event and other indicators. This function can reduce the nurses' processes for repeated transcription, saving time and avoiding the errors caused by transcription. The nursing information collection system provides a variety of nursing assessment sheets, post-operative nursing record sheets and the nursing record sheets for critically ill patients. Nurses can input the relevant information in the corresponding record sheets with PDA at the patient's bedside and the data are transmitted back to automatically generate the appropriate sheet. The information collection system through nursing monitoring sheets includes blood glucose monitoring sheet and multi-functional monitoring sheet, etc. The nurse can hold PDA at hand to scan the patient's wrist strap on the corresponding monitoring sheets, and after the verification, the results are monitored and input. Finally, the data are transmitted back to automatically generate the corresponding monitoring sheet.

Scanning of Barcodes for Patient's Test Specimens. Nurses can directly extract the information on medical orders for checking at the patient's bedside. Before the blood drawing, the nurse first uses PDA to scan the patient's barcodes respectively for test tube and wrist strap; after the confirmation, the nurse draws the patient's blood; and then after the completion of blood drawing, the nurse scan the barcode again. By doing so, the problem of mistaking the specimen at the source is solved thoroughly. 
Information System and Statistics. The intravenous infusion execution statistics system can be used to retrieve the information on nurses' medical order implementation at the PC terminal, including the persons operating at these links such as dosing, reviewing, infusion, inspection, infusion stop and the relevant operation time, providing reliable monitoring data for transfusion quality management. The nursing inspection statistics system can be used to make a statistics of the time for patient data collection, infusion inspection and ward visit and form a sheet for nursing inspections, solving the problem of no evidences for nurses' visits for patient.

\section{Advantages of Mobile Nursing Information System Applied in High Quality Nursing}

Move the nurses' workstation to the ward, improve the patients' satisfaction. According to the requirements for high-quality nursing services, the responsibility system and overall nursing should be implemented, proving the continuous whole-course nursing services for patients. In the service process, the nurses should carefully listen to the patients' feedbacks and opinions upon nursing, improving the patients' satisfaction continuously. Based on the technological application of wireless network, the PDA in mobile nurses' workstation moves the workplace of primary nurse to the ward through the real-time connection with the whole information system network. Such things as the infusion, liquid adding, tube sealing or nursing recording can be done through the mobile nursing station PDA. Through the infusion inspection module, the whole process of infusion can be recorded objectively in details, enhancing the nurse's awareness of initiative inspection, reducing and avoiding the occurrence of adverse transfusion and improving the safety of intravenous infusion. The measure of moving the nurses' workstation to the ward allows the primary nurse to have more time to provide bedside services and communication for patients while shortening the distance of nurses to the ward for visit and operation, effectively reducing the patient calls. Thus, the nurses can have more time to stay with patients and communicate with patients, and the patients can find their primary nurses at any time. The increase in the time of staying in the wards, the increase in the time of providing basic nursing for patients and the increase in the time of close contact with patients can be helpful in enhancing the nurse-patient communication, improving the nurse-patient relationship, increasing the confidence of patients in nurses and improving the patients' satisfaction.

Optimize the workflow of nurses, and enhance the initiatives of nurses. The nursing workflow can be optimized. Traditionally, when the nurses collect the data of physical signs and record the nursing process, they must transcribe a large amount of information for the second time. In this case, nurses spend a lot of efforts and time on the repeated transcription of information, increasing the probability of making errors as well as the nurses' workloads. Through the application of this system, the relevant date collected with PDA will be transmitted to the PC terminal to automatically generate the corresponding nursing sheets. As the nurses' workloads are reduced, the nurses have more time and efforts to pay attention to the patients, optimizing the nursing workflow and greatly improving the nurses' satisfaction.

Facilitate the performance appraisal, and improve the management level. Traditionally, as the nurses make the statistics of daily workloads manually, this process is tedious and prone to errors and is easily influenced by some human factors. After this system is applied, the computer can automatically make the statistics of the workload of each nurse, the number of critical patients nursed in the wards, the number of patients in grade one nursing and the number of specific nursing operations, facilitating the fair performance appraisal of departments and reasonable use of human resources. With this system, the head nurse can keep abreast of the nursing status of departments, and she/he can take the appropriate preventive measures against the problems found in the nursing process, making the remedial management after the incident become the preventive management before the incident. The mobile nursing information system enables the nursing quality control to be truly implemented at each link, achieving the transition from experience nursing management to scientific nursing management and making the nursing management more quantitative and scientific. 
Improve the quality and efficiency of clinical nursing. The application of PDA makes the nursing management more rigorous and standardized. In addition, it makes the nursing management to change a lot: from qualitative management to quantitative management, from the management by objectives to process management, from experience management to scientific management. Based on the data, it can be used to make the performance evaluations on individuals, departments and all the nursing work and reasonably allocate human resources. (1) In the process of using PDA, each patient must wear a unique wrist strap with the bar code containing basic information of patients and some important medical information, which can be used for the identification of the patient' $s$ identity when receiving various medical services, ensuring the accurate and efficient identity identification at the time of medical treatment. (2) Through PDA, the one-to-one correspondence between each medical order and actual operator can be formed; the time of medical order observation and the way of drug giving are recorded; the time of observing the patient's conditions and the relevant observed data are recorded and saved immediately. In this way, the behaviors of nurses can be regulated, and the reliable data for nursing can be provided, the case of confusion in responsibilities of implementing the medical orders can be avoided and the correctness and efficiency in nursing work can be improved. (3) In the application process of PDA, all data can be shared; the statistics of the amount of nursing from individuals, departments and the whole hospital can be made, providing the reliable reference basis for performance evaluation and allocation of human resources. The application of PDA, to a greater extent, promotes the continuous improvement of nursing quality, providing the basis for nursing managers to make the scientific decision and allowing the patients to have higher quality nursing services.

\section{Development Prospect and Summary}

The mobile nursing information system is of great significance to the scientific management of nursing work. It has been widely used in foreign countries, especially in the United States and other developed countries. With the rapid development of hospital information technology, the demands for clinical nursing information technology will grow gradually. The nursing information system, as the systematic software of clinical nursing work, will have considerably wide prospect in application. The nursing information system will develop towards the direction of nursing expert system, integrated management information system for hospital nursing and remote nursing. The development of mobile medical care is unstoppable, which has brought tremendous opportunities and challenges to wireless, networking, storage, security, software, integration and other fields.

At present, major hospitals in China have made great achievements in the nursing information system, but there are still many problems. Such problems as the lack of uniform standards for information entry for various units, difference in software development levels, nurses' weak awareness in use and unskilled operation can make the nursing information system fail in playing its proper role. Therefore, it is a necessary way of developing the nursing information system to enable the nurses to learn to distinguish and use effective information to solve problems at work through accelerating the standardization of nursing information system in establishment and enhancing the nurses' training on the knowledge and skills of nursing information system. I believe that, the standardized and efficient information system available in the near future will make the nursing work more scientific, rational and modernized.

\section{Acknowledgement}

In this paper, the research was sponsored by the Science and Technology project of education depart ment, heilongjiang province (Project No. 12531814). 


\section{References}

[1] Liu Lijie, Li Chun, Yan Jingdong. Application of Mobile Nursing Information System in the Demonstration Project Activity of Creating High-quality Nursing Services, J. Journal of Nursing. 2011, (09):23-24.

[2] Huang Meihong, Yu Wenmin, Zhang Xuemin, Xiao Xueqing. Development of Extending Funciton of Nursing Information System and Management, J. Journal of Nursing Science. 2012, (21):122-123.

[3] Li Sen, Wang Ling, Wu Xiaoying, Zhan Ying, Zhao Menglu. A Review on Application Effect Evaluation of Mobile Nursing Information System , J.Journal of Nursing Science. 2012, (22):237-238.

[4] Yan Xing yan, Ren Guohong. Design and Realization of Drug Supply Chain Management System , J.Chinese Medical Equipment Jounal. 2013, (05):6-8.

[5] Zhang Ying, Jia Xiaojun, Chi Yanyu, Xu Lijie. The Influencing Factors of Nurses' Use of Mobile Nursing Information System , J.Chinese Nursing Management. 2012, (12):77-78.

[6] Gao Fu, Tan Qiming. Experiences in the Application of PDA in the Improvement of Clinical Nursing Quality, J. Chinese Journal of Medical Device. 2011, (11):96-97.

[7] Sun Zhimei, Zhou Wenfang, Chen Jianping, Lin Ruijiao. Application of PDA in the Clinical Nursing Work , J. Journal of Fuzhou General Hospital. 2011, (03):58-59.

[8] Dong Mei, Yang Wenjun. Combined Application of Mobile Nursing Trolley and Terminal Control Computer in the Bedside Nursing, J.Chinese Journal of Nursing. 2013, (05):39-40. 